Brazilian Journal

of Chemical

Engineering

\title{
A NEW COMPUTATIONAL FLUID DYNAMICS STUDY OF A LIQUID-LIQUID HYDROCYCLONE IN THE TWO PHASE CASE FOR SEPARATION OF OIL DROPLETS AND WATER
}

\author{
Kh. Sharifi ${ }^{1}$, T. Jafari Behbahani ${ }^{1 *}$, S. Ebrahimi ${ }^{2}$, M. Sabeti ${ }^{3}$ and S. Soflaee ${ }^{4}$ \\ ${ }^{1}$ Research Institute of Petroleum Industry, Tehran, Iran. E-mail: jafarit@ripi.ir \\ 2 Islamic Azad University South Tehran Branch, Department of Management and Accounting, Tehran, Iran. \\ ${ }^{3}$ Isfahan University of Technology, Department of Chemical Engineering, Isfahan, Iran. \\ ${ }^{4}$ University of Sistan and Baluchestan, Department of chemical Engineering, Zahedan, Iran.
}

(Submitted: March 4, 2018 ; Revised: August 9, 2018 ; Accepted: April 1, 2019)

\begin{abstract}
A liquid-liquid hydrocyclone, so-called de-oiling equipment, was investigated for separation of oil droplets and wastewater in an Iranian petroleum unit. To generate the geometry and the mesh of the hydrocyclone Gambit software was used. Afterwards, by taking advantage of the Euler-Lagrangian principle, the governing equations covering the motion of fluid and that of particles in the hydrocyclone were solved. Initially, it was shown that the outcomes of CFD are in agreement with experimental data, and then 15 hydrocyclones with different overflow diameters, overflow lengths and input velocities were employed for more detailed analyses. The separation process through a hydrocyclone is counted as an economical process when ther is not only a low pressure drop inside the hydrocyclone, but also a high separation efficiency. As a result, regarding the mentioned criteria, the optimum hydrocyclone, whereby the highest performance of the hydrocyclone is exploited, was obtained by applying the 15 sets of CFD data to a Neural Network (NN) and then by optimizing the function generated by the NN by means of a Genetic Algorithm (GA). The innovation of this research work is the threephase modeling.

Keywords: Liquid-liquid hydrocyclone; Optimization; RSM turbulent model; CFD.
\end{abstract}

\section{INTRODUCTION}

Hydrocyclone as a two-phase separator has a widespread application in the oil and mining industry (Samaelli et al., 2017). The main feature of hydrocyclones is their simple installation, cheap operation, easy repair and high performance power. To get familiar with the separation efficiency and the velocity distribution inside hydrocyclones, several researchers have examined the performance of hydrocyclones in various circumstances, both numerically and experimentally. Liquid-liquid hydrocyclones are separators used for separation of dispersed oil and continuous fluid, usually regarded as water, in industrial units. A large number of researchers have investigated the swirl motion of fluids inside hydrocyclones from both the theoretical and the experimental point of view ( $\mathrm{Li}$ and Huang 2016; Huang, Li et al. 2017; Li and Huang 2017).

The CFD approach was used in various pieces of equipment in order to know clearly the properties of the equipment (Bayat et al., 2012; Khodabandeh et al., 2016; Samaelli et al., 2017; Khodabandeh et al., 2017; Khodabandeh et al., 2017; Mashayekhi et al., 2017; Sharifi et al., 2017). Because assembling hydrocyclones is not economically cheap and fast, researchers are more inclined to develop a CFD study into the velocity distribution of solid-liquid hydrocyclones or even liquid-liquid hydrocyclones. Grady et al. (2003) could determine the velocity

\footnotetext{
* Corresponding author: T. Jafari Behbahani - E-mail: jafarit@ripi.ir
} 
profile and separation efficiency in a $10-\mathrm{mm}$ liquidliquid hydrocyclone regarding the Reynolds Stress Model (RSM). The same was done by Paladino et al. (2005) using dispersed oil of very low density. In another study, an oil phase with high density was used by Huang (2005). The geometry of the hydrocyclone had two input nozzles at the opposite side of the equipment. The Eulerian-Eulerian approach was accompanied with RSM to observe the fluid motion of dispersed and continuous phases throughout the hydrocyclone. He proved that the geometry of the entrance leads to a change in the swirl motion of fluid. Chu et al. (2002), regarding a solid-liquid hydrocyclone, scrutinized the effects of the body and the inlet of the hydrocyclone on the separation efficiency. Energy losts in various parts of the hydrocyclone was the subject of their research. Hwang et al. (2008) investigated the effects of feed velocity and the overflow diameter which are attributed to a change in solid-liquid hydrocyclone performances. To do so, they used Rflow software built by Japan. The method they employed in the study was the Eulerian-Lagrangian approach. In fact, the approach of the trajectory of particle was implemented so that the number of solid particles exiting through the underflow part could be calculated. The supplementary description about the particle trajectory method and its influential forces is delineated in the next sections.

The role that some parameters, including the length of the overflow nozzle, the diameter of the overflow and feed velocity, play in a hydrocyclone was studied by prior researchers. However, there is no practical report gathering the effects of the mentioned parameters all together on hydrocyclone operation. The present work aims at analyzing the effects of the three parameters on a hydrocyclone and then finding the optimum point in which separation is conducted as best as possible. To this aim, Fluent as a CFD tool was employed to get results in various conditions, and then the programming software of Matlab was used to define and to optimize an objective function related to the results of CFD. The property of the fluid introduced into the hydrocyclone comes from the wastewater system of a NGL 1000 unit located in the south of Iran. Because the water treatment system of NGL 1000 faced a sever separation problem which caused the dispersed oil not to be differentiated from the outlet water, this work was presented to solve the treatment problem by using an appropriate hydrocyclone.

\section{SIMULATION OF THE FLOWING FLUID}

\section{Governing equations}

To see the required governing equations for the simulation, follow the equations below.
Continuity equation:

$\frac{\partial \rho}{\partial \mathrm{t}}+\nabla \cdot(\rho \overrightarrow{\mathrm{u}})=0$

Momentum equation:

$\frac{\partial(\rho \vec{v})}{\partial t}+\nabla \cdot(\rho \vec{u} \vec{u})=-\nabla P+\rho g+\vec{f}$

where $\mathrm{t}$ represents the time, $\rho$ is the symbol of the fluid density, $u$ shows the velocity of the fluid, $P$ is the pressure of the system and finally $f$ indicates the volume force exerted on the fluid.

The two governing equations have to be solved concomitantly in order for the velocity and pressure distribution of the fluid inside a hydrocyclone to be obtained. There are some assumptions taken into account, each of which is composed as follows:

a) The viscosity and the density of water are constant throughout the process.

b) The velocity near to the walls is zero.

c) At the whole cross section of the inlet, the velocity of fluid is $\mathrm{v}$.

d) The outflow boundary condition is set at the overflow and underflow .

The turbulent model of RSM is assumed to be dominated throughout the fluid motion (2017) (See Appendix for more information about RSM).

The Eulerian-Lagrangian approach is applied to the hydrocyclone system.

The particles dispersed in the primary fluid have spherical geometry.

\section{Particle trajectory theory}

The path that dispersed particles (droplets, sands or bubbles) follow comes from the balance of forces acting on a particle. The net force arising from Eq. (3) is equal to the particle inertia in the Cartesian coordinate along the $\mathrm{x}$-axis.

$\frac{d u_{p}}{d t}=F_{D}\left(u-u_{p}\right)+\frac{g_{x}\left(\rho_{p}-\rho\right)}{\rho_{p}}+F_{x}$

which $\mathrm{F}_{\mathrm{x}}$ is an additional acceleration term, and $F_{D}(u$ $\left.-u_{p}\right)$ represents the drag force exerted on the particle.

$F_{D}=\frac{18 \mu}{\rho_{p} d_{p}^{2}} \frac{C_{D} R e}{24}$

Herein, $u, u_{p}, \mu, \rho, \rho_{p}$ and $d_{p}$ are the fluid phase velocity, particle velocity, the viscosity of the fluid, the density of the fluid, the density of the particle and the particle diameter, respectively. The relative Reynolds number comes from the following equation. 
$\operatorname{Re}=\frac{\rho d_{p}\left|u-u_{p}\right|}{\mu}$

There exist some correlations to calculate the drag coefficient, $\mathrm{C}_{\mathrm{D}}$, in Eq. (4) in the Eulerian-Lagrangian reference frame. In the current manuscript, the drag coefficient is estimated through Eq. (6) . Eq. (6) says the drag coefficient changes with variation of $\mathrm{Re}$, while the geometry of the droplets, being spherical, remains constant throughout the process.

$\mathrm{C}_{\mathrm{d} \text {,shpere }}=\left\{\begin{array}{ll}0.424 & \operatorname{Re}>1000 \\ \frac{24}{\operatorname{Re}}\left(1+\frac{1}{6} \operatorname{Re}^{2 / 3}\right) & \operatorname{Re}<1000\end{array}\right\}$

In addition to the drag force, other forces are included in Eq. (3). These forces, which incorporate additional forces in the particle force balance, can be regarded as a significant term under special circumstances. For example, the virtual mass force and pressure gradient in the fluid, which are combined in Eq. (7), appear in Eq. (3).

$F_{x}=C_{v m} \frac{\rho}{\rho_{p}}\left(\overrightarrow{u_{p}} \nabla \vec{u}-\frac{d \overrightarrow{u_{p}}}{d t}\right)$

where $\mathrm{C}_{\mathrm{vm}}$ denotes the virtual mass coefficient which is set to 0.5 by default. Generally, when there is little difference between the densities of fluids, Eq. (7) sounds influential. Therefore, to calculate the performance of hydrocyclones, Eq. (7) should not be neglected when regarding hydrocarbons and water as the studied fluids.

\section{RESULTS AND DISCUSSION}

\section{Validation of the model}

To verify the accuracy of the present simulation, which includes solving the preceding equations via afinite volume method in a three-dimensional space, a really suitable meshing process is required. The hydrocyclone geometry chosen in the study comes from an experimental work developed by Bai et al. (2009). The size of each main part of the hydrocyclone is delineated in Fig 1 and Table 1. Whereas different authors have mostly applied tetrahedral cells to their geometries in order to mesh their hydrocyclones, a structured hexahedral mesh algorithm was employed throughout the whole hydrocyclone in the present work

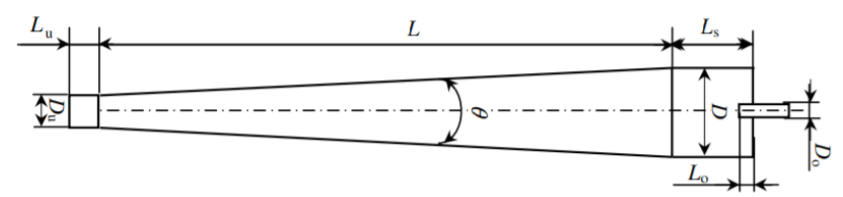

Figure 1. The overall view of the hydrocyclone whose geometry was analyzed in this study (Bai, et al., 2009).

(Motin et al., 2017), since this type of mesh produces more reliable results due to its logical aspect ratios and skewness. To illustrate it clearly, Fig 2 consisted of the geometry of the hydrocyclone and the type of meshes used is shown.

The whole of the body was divided into six separate sub-volumes, and each sub-volume in the hydrocyclone was separately meshed through Gambit 2.4. Two important commands of Map and Cooper in Gambit software which help establish a hexahedral mesh in each volume were used during the meshing procedure. Please note that selecting a proper meshing can contribute to a suitable convergence in solving the equations, whereas an improper one almost ends up with too much instability, divergence of calculation or even incorrect velocity/pressure profile production inside hydrocyclones. In this study an attempt was made to

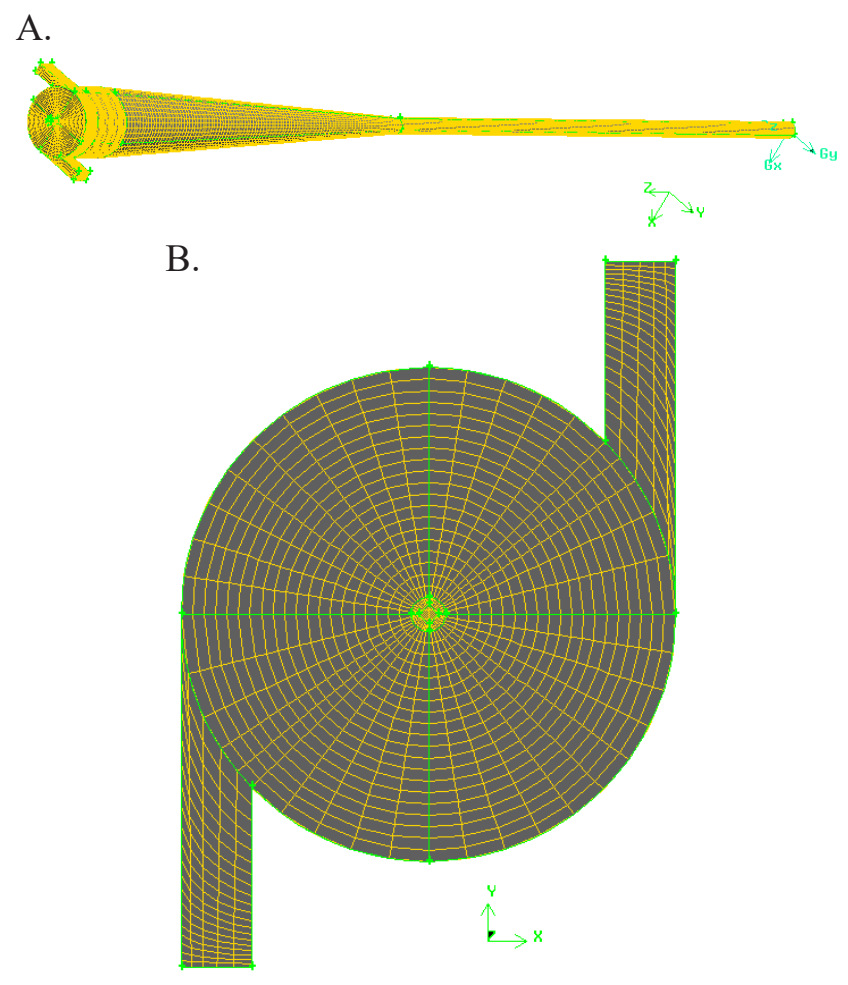

Figure 2. The hydrocylone geometry and the type of meshes used in this work. A) A 3D view of the hydrocyclone body. B) A top view of the hydrocyclone.

Table 1. The value of each edge shown in Fig 1. (D = 35mm).

\begin{tabular}{cccccc}
\hline $\mathbf{L s}$ & $\mathbf{D}_{\mathbf{0}}$ & $\mathbf{D}_{\mathbf{u}}$ & $\mathbf{L}_{\mathbf{0}}$ & $\mathbf{L}$ & $\mathbf{L}_{\mathbf{u}}$ \\
\hline $\mathrm{D}$ & $0.07 \mathrm{D}$ & $0.23 \mathrm{D}$ & $0.1 \mathrm{D}$ & $7.37 \mathrm{D}$ & $9.71 \mathrm{D}$ \\
\hline
\end{tabular}


produce a high quality of meshes with the skewness of 0.2 at most over the whole body of the hydrocyclone (except the inlet parts of the hydrocyclone). By doing so, the values of variables at the places where there exist sharp gradients could be caught more accurately.

As mentioned previously, the mixed fluid enters the hydrocyclone from the two inlet ports at the top of the hydrocyclone, then the fluid exits from the overflow and the underflow sections at the two ends of the hydrocyclone. To find the pressure and velocity field inside the hydrocyclone, the Semi-Implicit pressure Linked Equations (SIMPLE) method associated to the Fluent 15 (ANSYS, 2017) solver was used for simulation. The first order upwind discretization scheme for momentum was employed in order to determine the variables inside each cell. As the GreenGauss Node-Based method is the most accurate with respect to others, it was chosen for the present work to find the variables' gradient. Simulations were carried out until the value of the residual for variable continuity reached $1 \times 10^{-6}$. The computer used for this study was aan $i 7$ core processor with a $4 \mathrm{GHz} \mathrm{CPU}$ and 16 GB RAM memory. Upon reaching converged results under the steady state condition, the problem was switched to the unsteady one and run for several seconds to know the effects of turbulency on the hydrocyclone performance.

Fig 3 indicates the velocity distribution inside the studied hydrocyclone. This profile was for an inlet velocity of $4.16 \mathrm{~m} / \mathrm{s}$ of the fluid. The pressure drop between the inlet and the outlet below the hydrocyclone was computed via Fluent and is equal to $54 \mathrm{kPa}$. Initially, when entering the hydrocyclone, the fluid has horizontal swirl motion; afterwards, when volumes of the fluid gradually get away from the inlet sections, their swirl motions are changed into the downward vertical velocity through the underflow. In fact, the greater the distance from the inlet section, the higher the fluid volumes axial velocity because the velocity vectors in the $\mathrm{x}$ - and $\mathrm{y}$-direction change into the z-direction. The velocity on the surface of the hydrocyclone walls is zero since the no-slippage velocity was set as a boundary condition on the surface of the walls. However, due to its substantial turbulent characteristic, the downward velocity of the fluid grows suddenly just adjacent to the walls. Fig 4 clarifies this for the direction of the flowing fluid in the $x z$-plane $(y=0)$. After entering the conical section, the magnitude of the flowing fluid increases. It is an obvious fact that the greatest magnitude of the velocity is attributed to the center of the hydrocyclone (when the fluid is completely near to the underflow). Furthermore, while entering the upper section in the overflow, the flowing fluid could increase its velocity. The maximum velocities are attributed to the overflow and underflow with the velocities of 11.7 and $9.4 \mathrm{~m} / \mathrm{s}$,

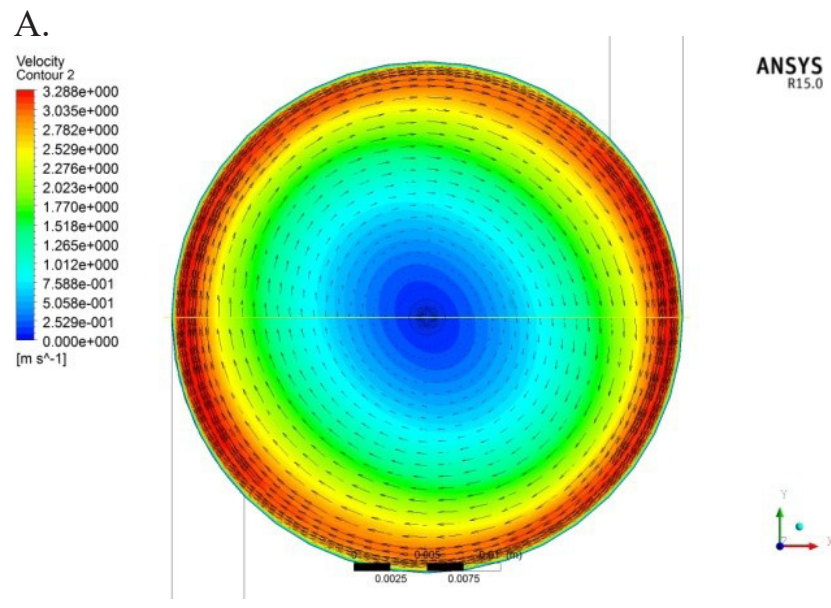

B.

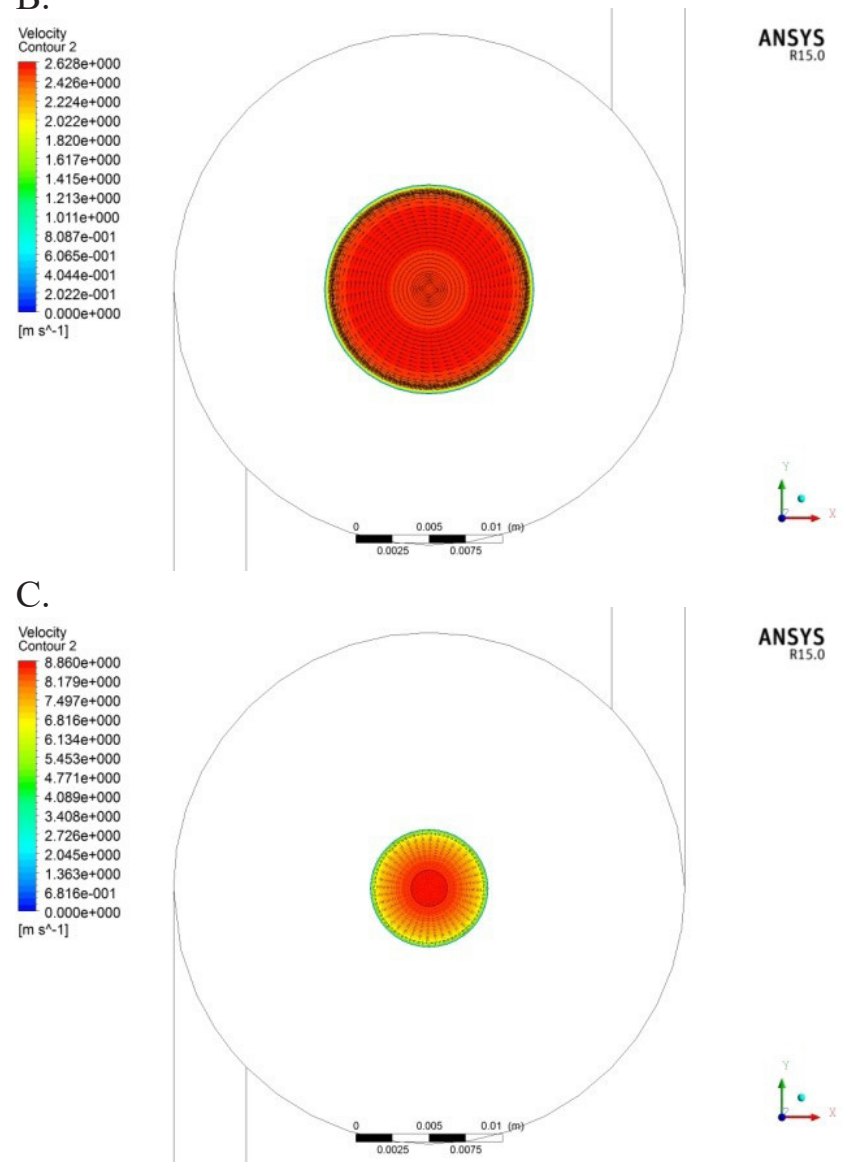

Figure 3. The velocity vectors and the contour diagrams of the axial velocity on the cross section of a) $Z=600 \mathrm{~mm} \mathrm{~b}) Z=300 \mathrm{~mm} \mathrm{c}) Z=100 \mathrm{~mm}$ in the $x-y$ plane.

of the same order. According to the figure, there is a surface around the center line where the magnitude of velocity is zero throughout. In this area, due to zero velocity, fluid as well as liquid particles do not go up or down. In the literature, this surface is called the locus of zero vertical velocity (LZVV). Similar to an interface, LZVV disconnects the space near to the center, where there is an upward velocity, and the space near to the walls, where there is downward 
A.

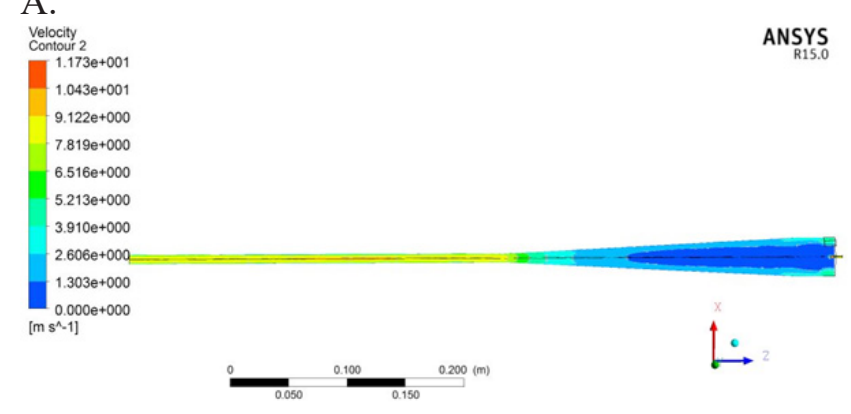

B.

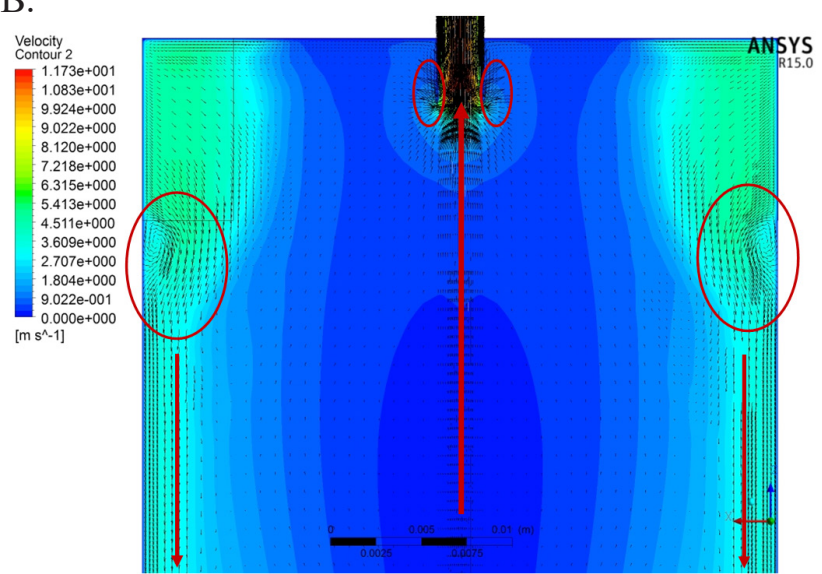

Figure 4. The contour plot for the velocity profile in the $x-z$ plane. A) an overall view of the hydrocyclone. B) the upper portion of the hydrocyclone. In the figure the velocity vectors at the whole of the portion were sketched. The red arrows indicate the flow direction in the center and near to the walls. Apart from that, the recirculation zones generated below the two entrance portions as well as near to the vortex finder are shown.

velocity. Therefore, there are two counter-current flows in the hydrocyclone. While the inside flow close to the centerline is called the secondary vortex flow, the outside flow is called the primary vortex flow. With the help of the secondary vortex flow, the oil droplets could ascend the vortex finder.

Apart from the two aforementioned vortex flows, there are some small circulations near to the inlet entrances as well as the vortex finder duct which are marked by bubble lines in Fig 4. From what was presented above, an oil droplet needs to pass the primary vortex flow in order to enter the secondary vortex flow and rise to the overflow. These small circulations may prevent the droplets from passing the primary space and entering the secondary vortex flow and lead to separation reduction.

To know the correctness of the model, the axial velocity of the model along the $x$-direction was validated against the experimental data. Fig 5 (a) compares the simulation with experiment. As stated above, the defined inlet velocity is $4.16 \mathrm{~m} / \mathrm{s}$ for both the experimental and CFD work. It is clear that, except where $\mathrm{r}<3 \mathrm{~mm}$, the numerical model could estimate all

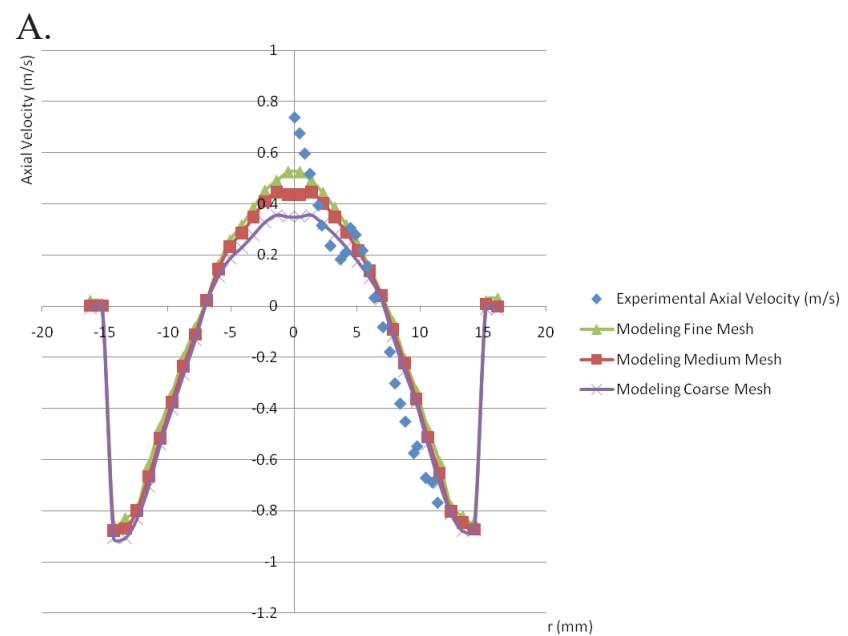

B.
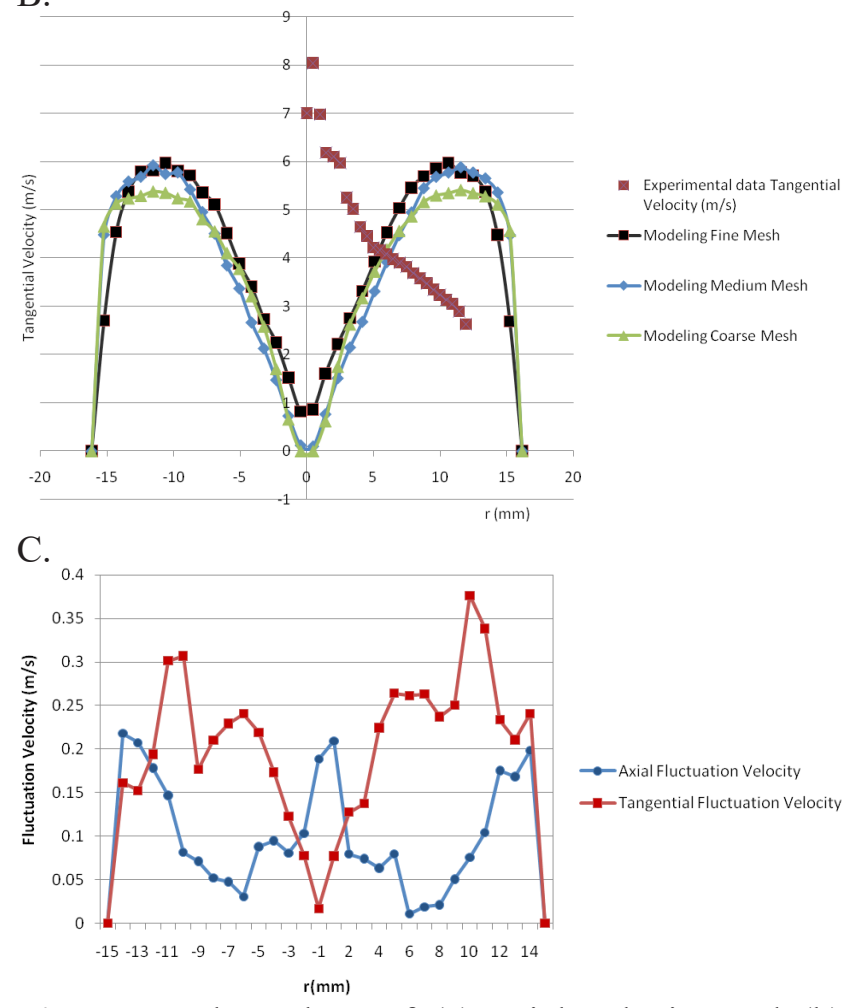

Figure 5. The values of (a) axial velocity and (b) tangential velocity along the $\mathrm{x}$-direction obtained from CFD results and experimental data c) the values of axial and tangential velocity along the $\mathrm{x}$-direction (modeling with a fine mesh).

of the velocity data accurately. To correctly understand the velocity distribution, various mesh numbers were examined so as to obtain more accurate data with Fluent software. The meshes were changed from low density to very high density by using Fluent tools. In fact, the first mesh had 250000 cells. Afterwards, the number of cells was increased to 450000 (the coarse mesh), 750000 (the medium mesh) and finally 1500000 (the fine mesh) by using Region and Gradient commands in Fluent software. The best results were assigned to the meshes with 750000 and 1500000 cells; 
however, to have not only an accurate analysis but also a fast computation, the mesh with 750000 cells in the hydrocyclone was chosen throughout the present study. In the following, the optimum conditions in which the hydrocyclone could work better are interpreted.

Despite having a good agreement between the experimental and the modeling results for the axial velocity, a discernible difference between the two results is detected when comparing the tangential velocities. The whole discrepancy taps into the overproduction of turbulence kinetic energy and turbulent viscosity in the RSM model. The length of the free vortex region linked to the turbulent viscosity increases in the RSM model due to capturing more anisotropy of flow and modeling the Reynolds stresses instead of using the eddy viscosity model (Saidi et al., 2012).

The axial and tangential fluctuating velocity profiles are described by the RMS of velocity fluctuations in the x-direction in Fig. 5 (c). Although the profiles do not have any smooth trends, it could be understood that the peaks in the axial fluctuation velocity diagram appear at locations where there are maximum axial velocities. This is also for the tangential fluctuation velocity diagram as well when comparing Fig. 5 (b) and Fig. 5 (c).

\section{Particle tracking}

After simulation and finding the important variables, such as the velocity distribution of the fluid inside the hydrocyclone, the path where the injected droplets move can be tracked via the particle trajectory process. All the properties, including the continuous fluid (wastewater) and the dispersed fluid (oil droplets), were taken from the NGL 1000 unit in Iran. The droplet particles were in the range of $1 \mu \mathrm{m}$ and $300 \mu \mathrm{m}$. The average density of the droplets during the process was assumed to be $800 \mathrm{~kg} / \mathrm{m}^{3}$. In addition, it was assumed that there was neither droplet-droplet nor droplet-fluid interaction between the two phases. The particle trajectory process can be conducted after finding the flowing fluid properties in Fluent. By doing so, those particles which escape from underflow are distinguished from those which are trapped in the overflow section. The efficiency of the separation process for the aforementioned particle sizes can be evaluated at different velocities. As an example, Fig. 6 demonstrates two types of droplets, one of which escapes from the underflow below and the other moves up toward the overflow.

By applying all of the above assumptions to the simulator, the separation efficiencies for the mentioned droplet sizes were calculated. Fig. 7 shows the hydrocyclone separation efficiency when the inlet velocity was set as $4.16 \mathrm{~m} / \mathrm{s}$. It was the inlet sections of the hydrocyclone where the droplets were injected. The

\section{A.}

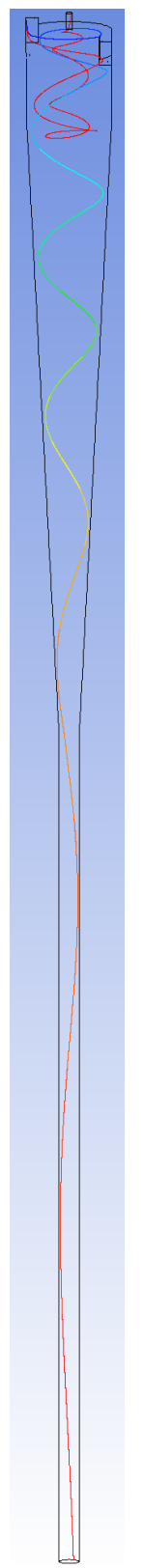

B.

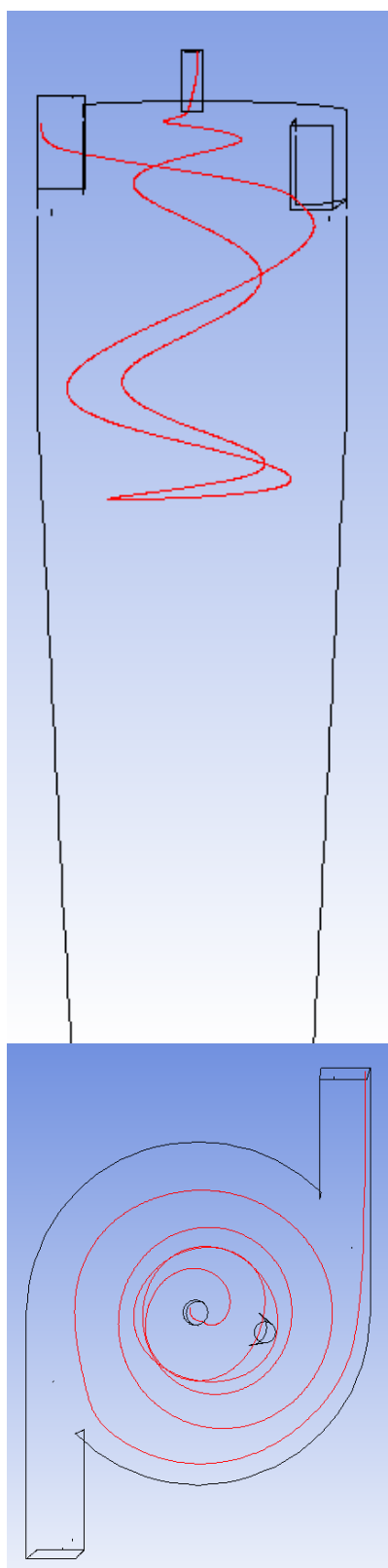

Figure 6. Two types of paths which particles follow in the hydrocyclone. A) the particle escapes when following this path (toward underflow) B) the particle is trapped when following the observed path (toward overflow).

droplet diameters change in size along the horizontal axis. Similar to what was stated in the theory section of the present manuscript, there exist three different kinds of force exerting pressure on each droplet in the liquid-liquid hydrocyclone. The net force coming from Eq. (3) determines the path where the particle has to track in the primary fluid. The force of gravity always pulls the droplet down. The two other forces determine whether the particle could pass the LZVV and move toward the secondary vortex flow or not. Although large particles are more inclined to drag outwards due to the drag force exerted on their large 
Different overflow lengthes

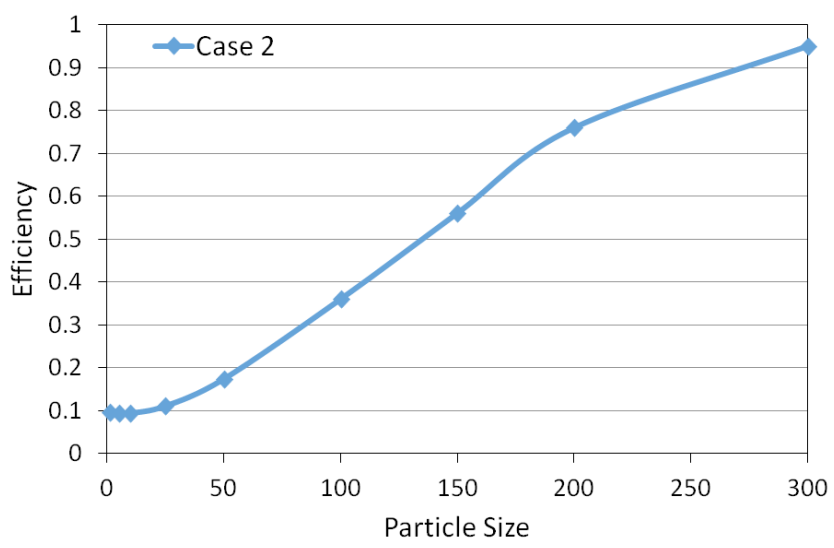

Figure 7. The hydrocyclone efficiency at various droplet sizes.

diameters, thanks to the additional forces they move inwards. In fact, the diameter of droplets determines whether the droplets could pass through the overflow or could escape from the underflow. From Fig 7, one can perceive that the larger the diameter of the droplet, the greater the chance the droplet can move through the overflow since the influence of additional forces, including the virtual mass force and pressure gradient, on the droplet is more effective. To summarize the expression, as shown in Fig. 7, an increase in droplet size increases the separation efficiency in the hydrocyclone.

\section{Performances of 15 cases}

In the following, fifteen types of hydrocyclones with various inlet diameters and overflow nozzles under three conditions of velocity were examined via Fluent software. Table 2 tabulates the parameters which were analyzed. The goal is to initially analyze and then to find the performances of the hydrocyclones during the separation process.

By applying the boundary and geometry conditions in Table 2 to the simulator, the velocity profile in the
Table 2. The studied hydrocyclones with various geometries and conditions. D denotes the diameter of the hydrocyclone.

\begin{tabular}{cccc}
\hline $\begin{array}{c}\text { Case } \\
\text { number }\end{array}$ & $\begin{array}{c}\text { Diameter } \\
\text { of overflow } \\
\mathbf{D}_{\mathbf{0}}(\mathbf{m m})\end{array}$ & $\begin{array}{c}\text { Overflow } \\
\text { length } \\
\mathbf{L}_{\mathbf{0}}(\mathbf{m m})\end{array}$ & $\begin{array}{c}\text { Inlet } \\
\text { velocity } \\
(\mathbf{m} / \mathbf{s})\end{array}$ \\
\hline 1 & $0.07 \mathrm{D}$ & $0.1 \mathrm{D}$ & 2 \\
2 & $0.07 \mathrm{D}$ & $0.1 \mathrm{D}$ & 4 \\
3 & $0.07 \mathrm{D}$ & $0.1 \mathrm{D}$ & 8 \\
4 & $0.07 \mathrm{D}$ & $0.2 \mathrm{D}$ & 2 \\
5 & $0.07 \mathrm{D}$ & $0.2 \mathrm{D}$ & 4 \\
6 & $0.07 \mathrm{D}$ & $0.2 \mathrm{D}$ & 8 \\
7 & $0.07 \mathrm{D}$ & $0.3 \mathrm{D}$ & 2 \\
8 & $0.07 \mathrm{D}$ & $0.3 \mathrm{D}$ & 4 \\
9 & $0.07 \mathrm{D}$ & $0.3 \mathrm{D}$ & 8 \\
10 & $0.14 \mathrm{D}$ & $0.1 \mathrm{D}$ & 2 \\
11 & $0.14 \mathrm{D}$ & $0.1 \mathrm{D}$ & 4 \\
12 & $0.14 \mathrm{D}$ & $0.1 \mathrm{D}$ & 8 \\
13 & $0.21 \mathrm{D}$ & $0.1 \mathrm{D}$ & 2 \\
14 & $0.21 \mathrm{D}$ & $0.1 \mathrm{D}$ & 4 \\
15 & $0.21 \mathrm{D}$ & $0.1 \mathrm{D}$ & 8 \\
\hline
\end{tabular}

hydrocyclone can be calculated. Table 3 summarizes the results of the liquid-liquid separation processes for the fifteen cases regarding a $150 \mu \mathrm{m}$-oil-droplet. Apart from the separation efficiencies, the rates of flow in both the overflow and underflow section, the values of split ratios, and the pressure drops in the fluid were calculated. The analyses of the calculated data are interpreted in the following sections.

\section{The effects of velocity on the separation efficiencies}

Comparing cases 1, 2 and 3 shown in Table 3 (and Fig. 8), one can understand the immense effect of the feed velocity on the separation efficiency in the hydrocyclone. When the velocity increases, the effects of both the drag and additional forces on the droplets become more important, and their powers outweigh the effect of the gravity force. Therefore, one would expect that more droplets (of the same size) could be trapped when the inlet velocity increases. For instance, while the separation efficiency for case

Table 3. The results of the separation of $150 \mu \mathrm{m}$-oil-droplet by using the hydrocyclones shown in Table 2.

\begin{tabular}{ccccccc}
\hline Case number & $\begin{array}{c}\text { Separation } \\
\text { efficiency }\end{array}$ & $\begin{array}{c}\text { Inlet flow } \\
\text { rate(kg/s) }\end{array}$ & $\begin{array}{c}\text { Overflow rate } \\
(\mathbf{k g} / \mathbf{s})\end{array}$ & $\begin{array}{c}\text { Underflow rate } \\
(\mathbf{k g} / \mathbf{s})\end{array}$ & $\begin{array}{c}\text { Split } \\
\text { ratio } \mathbf{( k g} / \mathbf{s})\end{array}$ & $\begin{array}{c}\text { Pressure drop } \\
(\mathbf{P a})\end{array}$ \\
\hline 1 & 0.21 & 0.19 & 0.01 & 0.18 & 0.91 & 13136 \\
2 & 0.56 & 0.41 & 0.03 & 0.37 & 0.91 & 54579 \\
3 & 0.78 & 0.79 & 0.06 & 0.73 & 0.91 & 193410 \\
4 & 0.29 & 0.19 & 0.01 & 0.18 & 0.91 & 13256 \\
5 & 0.47 & 0.41 & 0.03 & 0.38 & 0.91 & 54844 \\
6 & 0.73 & 0.79 & 0.06 & 0.73 & 0.91 & 192954 \\
7 & 0.27 & 0.19 & 0.01 & 0.18 & 0.92 & 12792 \\
8 & 0.53 & 0.41 & 0.03 & 0.37 & 0.91 & 57628 \\
9 & 0.79 & 0.79 & 0.06 & 0.73 & 0.91 & 194713 \\
10 & 0.44 & 0.19 & 0.05 & 0.14 & 0.70 & 9802 \\
11 & 0.65 & 0.41 & 0.11 & 0.29 & 0.71 & 40610 \\
12 & 0.78 & 0.79 & 0.21 & 0.58 & 0.72 & 143177 \\
13 & 0.57 & 0.19 & 0.09 & 0.10 & 0.54 & 6035 \\
15 & 0.76 & 0.41 & 0.22 & 0.19 & 0.46 & 25490 \\
\end{tabular}




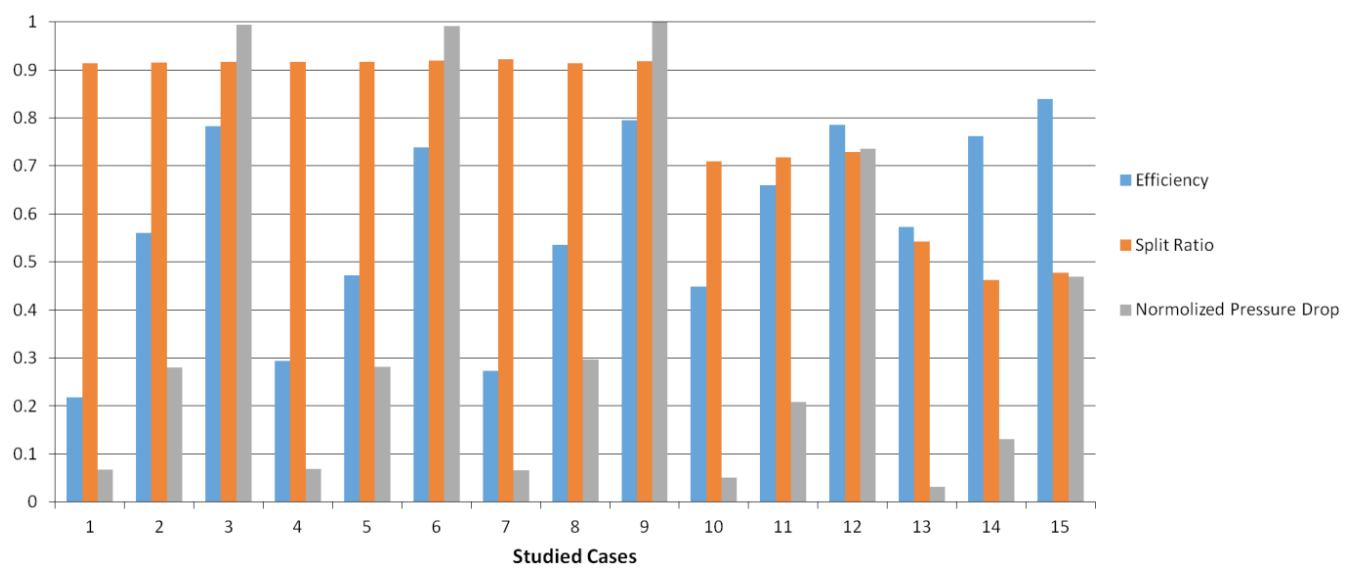

Figure 8. The investigation of the hydrocyclone performances in 15 cases categorized in Table 3.

3 is 0.8 , that for case 1 is equal to 0.2 . According to Table 3 , a change in the value of the inlet velocity does not lead to a significant change in the value of split ratio, while the pressure drop is largely increased with increasing velocity. From Fig. 8, one can perceive that the pressure drop in case 3 is ten times as big as that in case 1. A large amount of pressure drop culminates in a bad performance when using a hydrocyclone as a separator.

In addition, Fig 9 implies the fact that the velocity of the fluid does not have a significant effect on the separation of very small droplets, such as $1 \mu \mathrm{m}$, because of their small diameters. In other words, the two sources of drag and additional forces do not have enough strength to change the zone of droplets in the hydrocyclones from the primary vortex flow to the secondary vortex flow zone when the diameters are very small. When the diameter of droplets is increased to $5 \mu \mathrm{m}$, the two force sources are enhanced in value, and then the effect of the velocity of the flowing fluid grows tangibly. The differences between the separation efficiencies of droplets larger than $50 \mu \mathrm{m}$ are considerable in the three cases. This is followed by the effective impact of the velocity intensity over the swirl intensity and the generated turbulency (Schütz et al., 2009). By increasing the velocity magnitude,

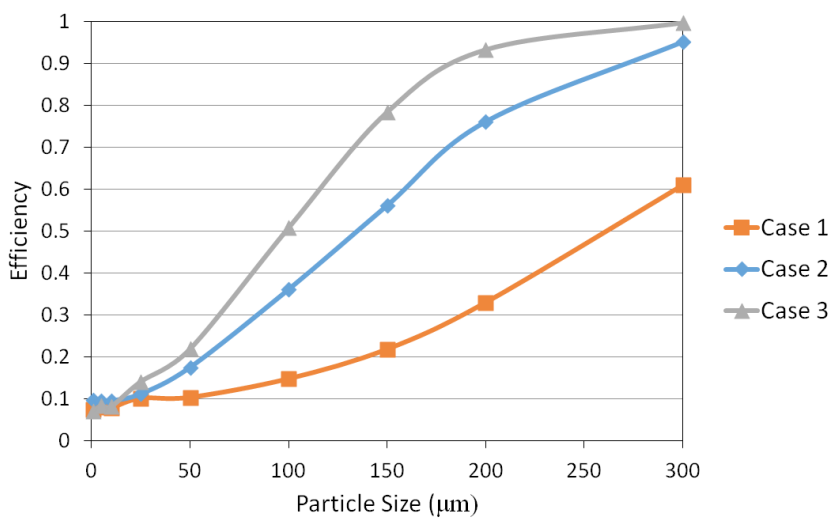

Figure 9. The effect of velocity on separation efficiency for cases 1,2 and 3 . the droplet size distributions in the figure shift a bit toward smaller droplets in comparison with the droplet size distributions at the lower velocity. Furthermore, according to Fig. 9, three curves eventually get closer until they somehow reach the same point. As a result, to eliminate a big droplet, such as $300 \mu \mathrm{m}$, in the water-oil mixture, the need of high velocity could be obviated. By reducing the flow rate, the pressure drop which is not desirable could be controlled.

In another study, regarding the constant velocity of $4 \mathrm{~m} / \mathrm{s}$, the efficiency of hydrocyclones (cases 2 , 5 and 8) with three different overflow nozzles was investigated. It was assumed that all other parameters remained without any change. Fig. 10 represents the change in the separation efficiency of droplets in the range of 1 to $300 \mu \mathrm{m}$ when using cases 2,5 and 8 . The three cases are almost the same. What comes to mind to elucidate this is the same axial velocities distributed in the center area of the hydrocyclones with each special nozzle length. Therefore, due to the small variation of axial velocities at the mentioned heights (the nozzle lengths), no big differences arise from the three studied cases. Although there is no large discrepancy between the three diagrams, case 2 has the best performance in the whole droplet range. According to Fig 8, one can perceive that these three cases even have the same

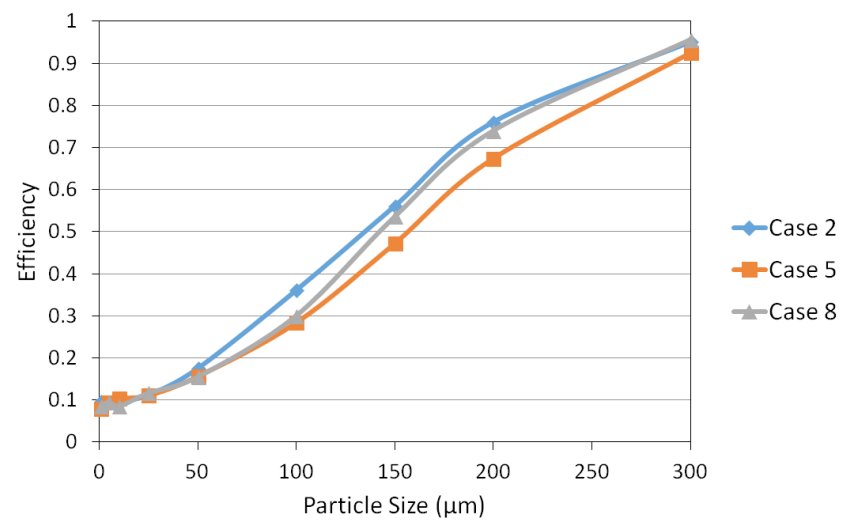

Figure 10. The effect of the variation of overflow nozzle lengths on the separation efficiency. 
pressure drop and split ratio during their operation. Given this, compared to the two other parameters, overflow nozzle length does not play a significant role in the separation process.

The need to obtain a suitable diameter for the overflow is an essential issue to design a proper hydrocyclone for industrial field application. To reach this aim, three hydrocyclones which only have a different overflow diameter were chosen for more studies. These hydrocyclones are case 2, 11 and 14. Fig. 11 shows the effect of the overflow diameter on the separation efficiency. When enlarging the overflow orifice size, the amount of water present in the secondary vortex flow is increased, so more droplets varied in size find opportunity to ascend toward the overflow when the overflow size is bigger (Kharoua et al., 2010). This can clearly been seen from the figure. The biggest discrepancy for the separation efficiency is attributed to the droplet of $50 \mu \mathrm{m}$. The effect of the diameter can be neglected when the diameters of droplets are more than $200 \mu \mathrm{m}$. Although the separation efficiency is increased upon enlarging the overflow diameter, the amount of water lost is increased due to its low split ratio. According to Table 3, when using case 4 , the split ratio reached its lowest value, 0.46. Thus, the overflow with large diameter almost does not end up with the best performance. To summarize the main point, to separate big droplets, a hydrocyclone with large diameter does not necessarily suit.

A hydrocyclone suits our needs when it has not only high separation efficiency but also a high split

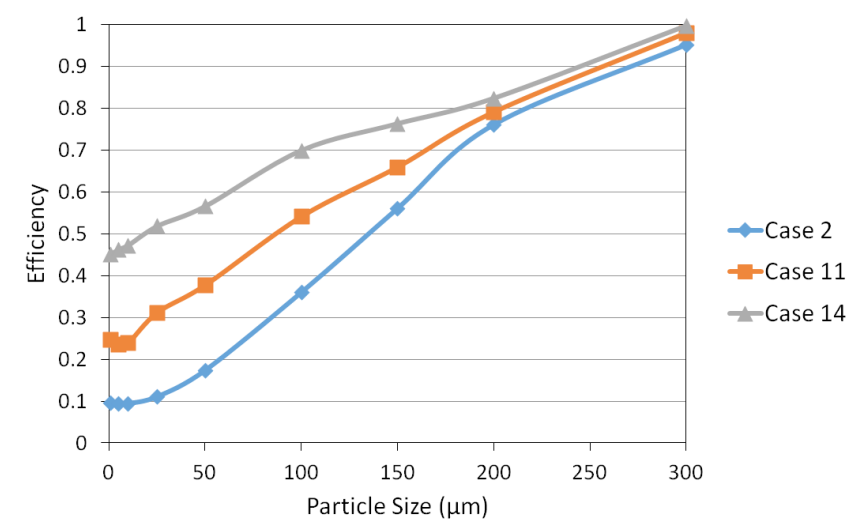

Figure 11. The effect of the overflow diameter on the separation. Three hydrocyclones are cases 2,11 and 14. ratio along with a low pressure drop. Regarding these matters, designers should generate successful hydrocyclones. By looking at Fig. 8, one could roughly state that hydrocyclone 11 (case 11) could be the best choice. However, to scientifically design the best hydrocyclone, there is a need of an optimum point which engineers could use according to their requirements. Herein, this was done with the help of Genetic Algorithm (GA) and Artificial Neural Network (ANN) tools together. Initially, an ANN was used to prepare a continuous function, and then, using GA, the best geometry and inlet velocity which lead to the best hydrocyclone performance were obtained.

The input variables in ANN are the inlet velocity, the diameter of overflow and the length of the overflow nozzle. To train the ANN, the data in Table 3 were used. Eleven cases were called training data, whereas the rest of them were counted as test data. The test data, which were chosen randomly, are responsible for testing the ability of the NN to show whether its accuracy is high enough to estimate the values of separation efficiency, split ratio and pressure drop in the flowing fluid correctly or not. Several NNs with different neurons and layers were tested in MATLAB software in order to generate a proper $\mathrm{NN}$ frame which had the lowest average absolute relative error (AARE) as well as a correlation coefficient $\left(\mathrm{R}^{2}\right)$ near to one.

Table 4 shows the results of different $\mathrm{NN}$ configurations for predicting the CFD outcomes. From the table, the NN with a 3333 configuration is the best frame having the lowest error. This configuration consists of four layers. The first layer receives three input variables and the last one generates three outputs as the results of the NN. There are also two hidden layers, each of which are made up of three neurons. To determine the best configuration, not only was AARE considered, but also $\mathrm{R}^{2}$ was the subject of the choice.

Fig. 12 shows the ability of the neural network with the 3333 configuration to predict the CFD outputs. As seen in Fig 12, the developed network could predict the outcomes extracted from CFD successfully. While the separation efficiency prediction (in Fig 12 (a)) falls within error bands of $\pm 15 \%$, the predictions of split ratios and pressure drops (in Fig 12 (b) and (c)) are within error bands of $\pm 10 \%$. These figures state that the 3333 configuration is the desirable frame which we could rely on to find our proper hydrocyclone.

Table 4. The validation of different NN configurations by taking advantage of AARE and R2.

\begin{tabular}{cccccc}
\hline Case & NN configuration & AARE train & R2 train & AARE test & R2 test \\
\hline 1 & 3223 & 3.76 & 0.99 & 11.44 & 0.54 \\
2 & 3323 & 8.67 & 0.92 & 6.51 & 0.48 \\
3 & 3333 & 3.76 & 0.99 & 0.99 & 0.92 \\
4 & 333 & 3.76 & 0.99 & 6.76 & 0.70 \\
5 & 343 & 3.76 & 0.99 & 6.40 & 0.90 \\
6 & 353 & 3.76 & 0.99 & 8.30 & 0.91 \\
7 & 363 & 3.76 & & & 0.83 \\
\hline
\end{tabular}



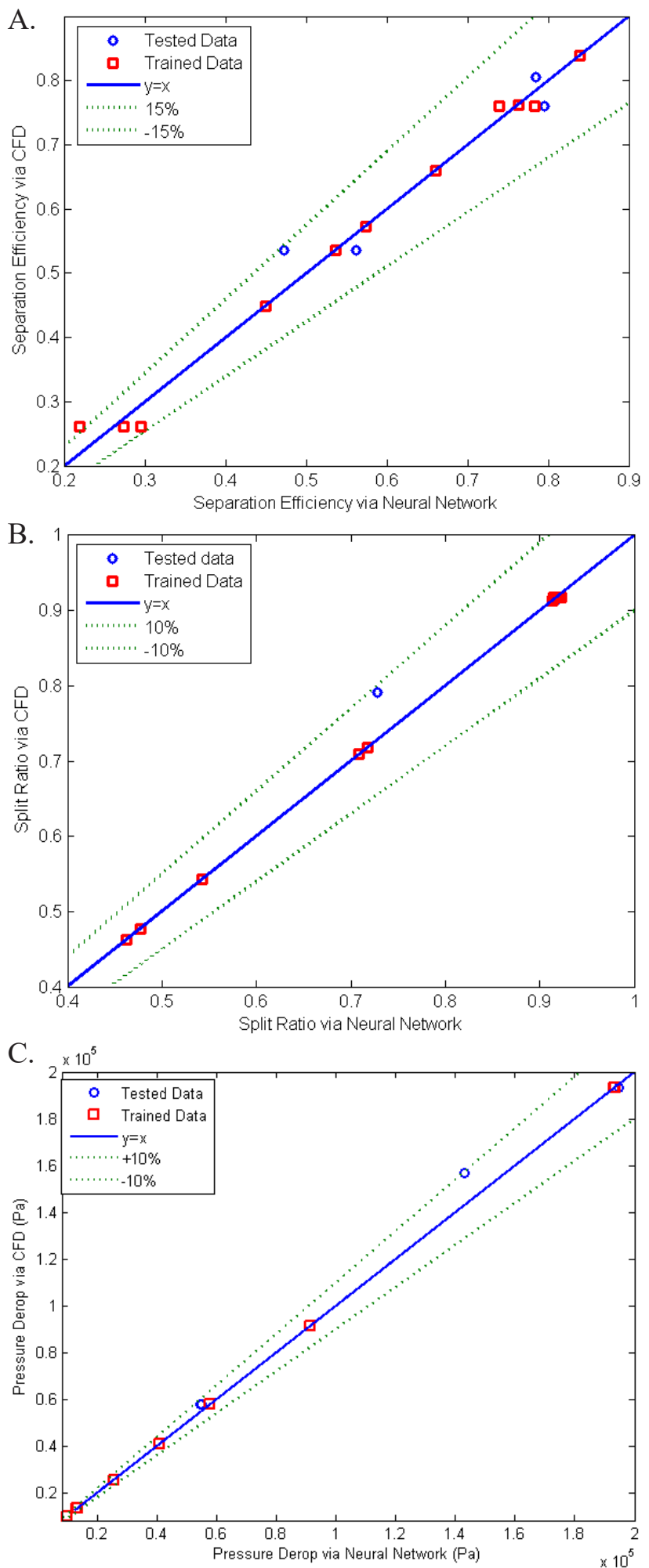

Figure 12. Validation of the neural network and CFD results. A) The ability of the neural network to predict separation efficiencies. B) The ability of the neural network to predict the split ratios of the hydrocyclones. C) The ability of the neural network to predict the pressure drops of the hydrocyclones.

According to the aforementioned expressions, now that the available criteria show that the suggested NN is able to generate accurate separation efficiencies, split ratios and pressure drops, the optimum point which includes the desired inlet velocity, overflow diameter and nozzle length could be computed via GA. The function created by NN was applied to GA in MATLAB software. The body of GA was made up of 100 generations, 100 populations with a relative error of $10^{-6}$ at most. The GA was run until its outcomes satisfied the mentioned options. According to the GA results, the inlet velocity of $3.5286 \mathrm{~m} / \mathrm{s}$, the overflow diameter of $0.2072 \mathrm{D}$ and the overflow length of 1D were attributed to the optimum point which produces the maximum efficiency from different aspects in terms of separation efficiencies, split ratios and pressure drops all together. This point results in the separation efficiency of 0.61 , the split ratio of 0.7077 and the pressure drop of $30 \mathrm{kPa}$.

\section{CONCLUSION}

The innovation of this research work is the threephase modeling. Finding the optimum point for which a reliable hydrocyclone could be designed and operated in order that an Iranian refinery could get rid of dispersed oil in its wastewater system was the aim of this study. The results showed that the higher the inlet velocity of the hydrocyclone, the higher the swirl motion and the better separation efficiency the hydrocyclone produces. However, a very high velocity results in a very high pressure drop in the discharged fluid. It was postulated that, when the size of droplets are either very small or very large, the inlet velocity does not have a significant effect on the separation efficiency. In addition, it was shown that, with increasing the overflow diameter, the number of droplets trapping in the overflow section is increased. Nonetheless, an increase of the diameter inevitably leads to more discharge of the primary fluid from the overflow. Furthermore, on the condition that the droplet sizes are larger than $150 \mu \mathrm{m}$, the diameter of the overflow could be counted as a negligible parameter in the separation process. Aside from that, how the length of overflow nozzle changes the separation efficiency was another aspect of this study. To seek the best performance, the outcomes of CFD were applied to an ANN. Afterwards, using GA, the optimum points for the inlet velocity, $3.5226 \mathrm{~m} / \mathrm{s}$, and the overflow diameter, 0.2072D, and the overflow length, $1 \mathrm{D}$, were computed.

\section{REFERENCES}

ANSYS. Modeling Turbulence, Theory Guide, ANSYS FLUENT 18.0 Documentation (2017).

Bai, Z. S., Wang, H. L., Tu, S. T. Experimental study of flow patterns in deoiling hydrocyclone. Minerals Engineering, 22, 319-323 (2009). https://doi. org/10.1016/j.mineng.2008.09.003 
Bayat, M., Aminian, J., Bazmi, M. Shahhosseini, S., Sharifi, K. CFD modeling of fouling in crude oil pre-heaters. Energy Conversion and Management, 64, 344-350 (2012). https://doi. org/10.1016/j.enconman.2012.05.003

Chu, L. Y., Chen, W. M., Lee, X. Z. Effects of geometric and operating parameters and feed characters on the motion of solid particles in hydrocyclones. Separation and purification Technology, 26, 237-246 (2002). https://doi. org/10.1016/S1383-5866(01)00171-X

Grady, S. A., Wesson, G. D., Abdullah, M., Kalu, E. E. Prediction of 10-mm hydrocyclone separation efficiency using computational fluid dynamics. Filtration \& Separation, 40, 41-46(2003). https://doi.org/10.1016/S0015-1882(03)00930-3

Huang, Q., Li, C. A mathematical model for predicting oil-water separation efficiency in a de-oiling hydrocyclone. Journal of Dispersion Science and Technology, 38, 1319-1324 (2017). https://doi.org $/ 10.1080 / 01932691.2016 .1236266$

Huang, S. Numerical Simulation of Oil-water Hydrocyclone Using Reynolds-Stress Model for Eulerian Multiphase Flows. The Canadian Journal of Chemical Engineering, 83, 829-834(2005). https://doi.org/10.1002/cjce.5450830504

Hwang, K.-J., Wu, W.-H.. CFD study on the effect of hydrocyclone structure on the separation efficiency of fine particles. Separation Science and Technology, 43, 3777-3797 (2008). https://doi. org/10.1080/01496390802286637

Kharoua, N., Khezzar, L. Hydrocyclones for deoiling applications - a review. Petroleum Science and Technology, 28, 738-755(2010). https://doi. org/10.1080/10916460902804721

Khodabandeh, E., Ghaderi, M. Parametric Study of Heat Transfer in an Electric Arc Furnace and Cooling System. Applied Thermal Engineering, 123, 1190-1200 (2017). https://doi.org/10.1016/j. applthermaleng.2017.05.193

Khodabandeh, E., Pourramezan, M. Effects of excess air and preheating on the flow pattern and efficiency of the radiative section of a fired heater. Applied Thermal Engineering, 105, 537-548 (2016). https:// doi.org/10.1016/j.applthermaleng.2016.03.038

Khodabandeh, E., Rahbari, A. Experimental and numerical investigations on heat transfer of a water-cooled lance for blowing oxidizing gas in an electrical arc furnace. Energy Conversion and Management, 148, 43-56(2017). https://doi. org/10.1016/j.enconman.2017.05.057

Li, C., Huang, Q. Rheology-Based Computational Fluid Dynamics Modeling for De-oiling Hydrocyclone Efficiency. Chemical Engineering \& Technology, 39, 899-908 (2016). https://doi. org/10.1002/ceat.201500623
Li, C., Huang Q. Analysis of droplet behavior in a deoiling hydrocyclone. Journal of Dispersion Science and Technology, 38, 317-327 (2017). https://doi.or $\mathrm{g} / 10.1080 / 01932691.2016 .1164062$

Mashayekhi, R., Khodabandeh, E. Application of a novel conical strip insert to improve the efficacy of water - Ag nanofluid for utilization in thermal systems: A two-phase simulation. Energy Conversion and Management, 151, 573-586 (2017). https://doi.org/10.1016/j.enconman.2017.09.025

Motin, A., Tarabara, V. V. Hydrodynamics within flooded hydrocyclones during excursion in the feed rate: Understanding of turndown ratio. Separation and Purification Technology, 185, 41-53 (2017). https://doi.org/10.1016/j.seppur.2017.05.015

Noroozi, S., Hashemabadi, S. H. CFD simulation of inlet design effect on deoiling hydrocyclone separation efficiency. Chemical Engineering \& Technology, 32, 1885-1893 (2009). https://doi. org/10.1002/ceat.200900129

Paladino, E. E., Nunes, G. CFD analysis of the transient flow in a low-oil concentration hydrocyclone. Conference Proceedings of the AIChE Annual Meeting (2005).

Saidi, M., Maddahian, R. Modeling of flow field and separation efficiency of a deoiling hydrocyclone using large eddy simulation. International Journal of Mineral Processing, 112, 84-93 (2012). https:// doi.org/10.1016/j.minpro.2012.06.002

Samaeili, M., Hashemi, J., Sabeti, M., Sharifi, K. Modeling and Analyzing Hydrocyclone Performances. Iranian Journal of Chemistry and Chemical Engineering, 36, 177-190 (2017).

Schütz, S., Gorbach, G. Modeling fluid behavior and droplet interactions during liquid-liquid separation in hydrocyclones. Chemical Engineering Science, 64, 3935-3952 (2009). https://doi.org/10.1016/j. ces.2009.04.046

Sharifi, K., Sabeti, M. Computational Fluid Dynamics (CFD) Technique to Study the Effects of Helical Wire Inserts on Heat Transfer and Pressure Drop in a Double Pipe Heat Exchanger. Applied Thermal Engineering, 128, 898-910 (2017). https://doi. org/10.1016/j.applthermaleng.2017.08.146

\section{APPENDIX}

The Reynolds stress model is one of the turbulence models based on Reynolds-averaged Navier-Stokes (RANS) equations (Noroozi and Hashemabadi 2009). It has greater potential to accurately predict complex flows since the model accounts for the effects of streamline curvature, eddy, swirl, and quick changes in stress rate in a more rigorous manner.

In the RSM, the eddy viscosity approach is discarded and the Reynolds stress components $\left(R_{k, i j}\right)$ 
are calculated directly. The turbulence stress tensor $\left(\tau_{k}^{t}\right)$ can be defined as:

$\tau_{\mathrm{k}}^{\mathrm{t}}=-\rho_{\mathrm{k}} R_{\mathrm{k}, \mathrm{ij}}$

$\mathrm{R}_{\mathrm{k}, \mathrm{j}}=\tilde{\mathrm{u}}_{\mathrm{k}, \mathrm{i}} \tilde{\mathrm{u}}_{\mathrm{k}, \mathrm{j}}$

The turbulence stress components $\left(R_{k, i j}\right)$ can be obtained from the following partial differential equation:

$\frac{\partial}{\partial t}\left(\bar{a} \rho R_{i j}\right)+\frac{\partial}{\partial x_{1}}\left(\bar{a} \rho \bar{u}_{1} R_{i j}\right)=P_{i j}+D_{i j}+\Phi_{i j}-\varepsilon_{i j}$

where $P_{i j}$ is the production of turbulence by the mean strain and can be expressed by the formulation:

$P_{i j}=-\bar{a} \rho\left(R_{i l} \frac{\partial \bar{u}_{j}}{\partial x_{1}}+R_{j l} \frac{\partial \bar{u}_{i}}{\partial x_{1}}\right)$

The diffusion term $\left(D_{i j}\right)$, which consists of the molecular diffusion $\left(D_{L i j}\right)$ and turbulent diffusion $\left(D_{T i j}\right)$, can be written as:

$\mathrm{D}_{\mathrm{ij}}=\mathrm{D}_{\mathrm{Lij}}+\mathrm{D}_{\mathrm{Tij}}=\frac{\partial}{\partial \mathrm{x}_{1}}\left(\overline{\mathrm{a}} \mu \frac{\partial \mathrm{R}_{\mathrm{ij}}}{\partial \mathrm{x}_{1}}\right)+\mathrm{C}_{\mu} \frac{\partial}{\partial \mathrm{x}_{1}}\left(\overline{\mathrm{a}} \frac{\mu_{\mathrm{t}}}{\sigma_{\mathrm{k}}} \frac{\partial \mathrm{R}_{\mathrm{ij}}}{\partial \mathrm{x}_{1}}\right)(\mathrm{A}-4)$

The turbulence viscosity $\mu_{t}$ can be calculated by:

$\mu_{\mathrm{t}}=\rho \mathrm{C}_{\mathrm{s}} \frac{\mathrm{K}^{2}}{\varepsilon}$

$\mathrm{K}=\frac{1}{2} \mathrm{R}_{\mathrm{ij}}$
Using the Kolmogrov assumption of local isotropy, the dissipation rate tensor $\left(\varepsilon_{i j}\right)$ can be expressed as:

$\varepsilon_{\mathrm{ij}}=\frac{2}{3} \varepsilon \overline{\mathrm{a}} \rho \delta_{\mathrm{ij}}$

where $\varepsilon$ is the scalar turbulent dissipation rate and is given by:

$\frac{\partial\left(\rho \overline{\mathrm{u}}_{\mathrm{u}} \varepsilon\right)}{\partial \mathrm{x}_{1}}=\frac{\partial}{\partial \mathrm{x}_{1}}\left(\mathrm{C}_{\varepsilon} \rho \frac{\varepsilon}{\mathrm{K}} \mathrm{R}_{\mathrm{ij}} \frac{\partial \varepsilon}{\partial \mathrm{x}_{\mathrm{j}}}\right)+\frac{\varepsilon}{\mathrm{K}}\left(-2 \mathrm{C}_{\varepsilon 1} \rho \mathrm{R}_{\mathrm{ij}} \frac{\partial \overline{\mathrm{\mu}}_{\mathrm{i}}}{\partial \mathrm{x}_{1}}-\mathrm{C}_{\varepsilon 2} \rho \varepsilon\right)(\mathrm{A}-7)$

$\phi_{i j}$ is the redistribution of turbulence by the pressure strain. The pressure strain correlation plays an important role in the Reynold stress model. For modeling the pressure strain different alternatives have been presented in the literature. In this work, the linear pressure strain correlation was used.

$\phi_{\mathrm{ij}}=\phi_{\mathrm{ij} 1}+\phi_{\mathrm{ij} 2}$

and,

$\phi_{\mathrm{ij} 1}=-\mathrm{C}_{1} \rho \frac{\varepsilon}{\mathrm{K}}\left(\mathrm{R}_{\mathrm{ij}}-\frac{2}{3} \delta_{\mathrm{ij}} \mathrm{K}\right)$

where the eight constants in the RSM equations are:

$\mathrm{C}_{\mu}=0.24, \mathrm{C}_{1}=1.8, \mathrm{C}_{2}=0.6, \mathrm{C}_{\varepsilon 1}=1.44$,

$\mathrm{C}_{\varepsilon 2}=1.92, \mathrm{C}_{\varepsilon}=0.13, \delta_{\mathrm{k}}=0.82, \mathrm{C}_{\mathrm{s}}=0.09$

Thus, the Reynolds stress turbulence model is formulated with six partial differential equations of the Reynolds stress tensor components and one equation for the scalar turbulent dissipation rate, in total seven partial differential equations. 